\title{
Intérêt et limites des mesures thermographiques microondes pour le diagnostic et le pronostic des irradiations aiguës localisées chez le porc*
}

\author{
F. DABURON, J.L. LEFAIX, J. REMY, G. FAYART, Y. TRICAUD** \\ (Manuscrit recu le 13 mai 1985)
}

\begin{abstract}
RÉSUMÉ
Des mesures thermographiques microondes ont été réalisées sur 30 porcs irradiés localement sur une cuisse, avec une source collimatée d'iridium 192 et à des doses variant entre 30 et 84 Gy (dose à $2 \mathrm{~cm}$ de profondeur).

Les méthodes de mesure et de traitement des résultats ont été développées selon un aspect qualitatif (rapports entre les images thermographiques et l'évolution anatomo-clinique des lésions) et un aspect quantitatif qui tente d'utiliser des index calculés à partir des données thermographiques brutes pour évaluer les relations entre l'intensité de la réaction thermique locale et la dose ou le débit de dose appliqués.
\end{abstract}

A côté de la valeur diagnostique de la méthode, qui rend mieux compte des atteintes radiologiques globales que de la dose physique réellement distribuée, son aspect pronostique, beaucoup plus fiable, permet très précocement (entre 10 et 15 jours) d'apprécier la gravité des lésions et leur évolution ultérieure.

\section{ABSTRACT}

Microwaves thermographic measurements were carried out on 30 pigs following local exposure of the thigh by a collimated source of iridium 192 at doses ranging between 30 and $84 \mathrm{~Gy}(2 \mathrm{~cm}$ depth dose). Measurement and data processing methods were developed both qualitatively (thermographic images vs anatomo-clinical evolution of the lesions) and quantitatively (by means of indexes calculated from the rough thermographic data to evaluate the relations between the intensity of the local thermic reaction and the applied dose or dose rate).

Beside the diagnostic value of the method, which accounts for the global radiological injuries better than the physical dose really distributed, its pronostic value - much more reliable - makes it possible to estimate the severity of the injuries and their later evolution very early (between 10 and 15 days).

* Ce travail a bénéficié d'un contrat AIEA n³731/TC.

** Commissariat à l'énergie atomique, Institut de protection et de sûreté nucléaire (IPSN), Département de protection sanitaire, Laboratoire de radiobiologie appliquée, INRA, 78350 Jouy-en-Josas. 


\section{INTRODUCTION - POSITION DU PROBLEME}

Le développement dans l'industrie métallurgique des techniques de radiographie (gammagraphie industrielle) utilisant de puissantes sources de rayonnement a multiplié, ces dix dernières années, les cas d'irradiation locale aiguë chez l'homme [14] ; quelques cas d'exposition à partir d'irradiateurs à usage médical ont été aussi décrits.

II s'agit, pour ce type de lésions, de réaliser une exploration non traumatisante de la zone irradiée - ce qui exclut les biopsies et les injections répétées d'indicateurs marquées - pour déterminer d'une part son étendue et d'autre part l'intensité de l'atteinte radiologique, ces deux paramètres étant l'expression directe de la répartition de la dose dans les tissus superficiels et profonds.

La thermographie infrarouge donne une bonne indication de l'émission thermique des couches superficielles de l'épiderme et, dans une certaine mesure, de la dose recue par une relation de la forme " à chaque isotherme correspond une isodose" ; mais on n'a aucune information sur la répartition de la dose en profondeur qui dépend de l'énergie du rayonnement de la source, et de la distance source-peau (facteur souvent difficile à apprécier dans les reconstitutions d'accidents et qui souvent aussi a varié au cours de l'exposition) [13].

La thermographie microondes n'est appliquée aux tissus vivants que depuis $1974[7,10,11]$; elle permet des mesures jusqu'à des profondeurs variables, selon la nature des tissus et la fréquence utilisée pour laquelle le capteur est accordé [16] : $0,5 \mathrm{~cm}$ à $9 \mathrm{GHz}, 2 \mathrm{~cm}$ à $3 \mathrm{GHz}$ et $4 \mathrm{~cm}$ à $1 \mathrm{GHz}$.

En ce qui concerne le choix de l'animal d'expérience, le porc semble un bon modèle pour une éventuelle extrapolation des observations à I'homme. En effet, la peau du porc présente de nombreuses analogies avec celle de l'homme [15] ; son volume corporel permet de procéder à des irradiations sur des surfaces importantes et d'étudier les effets en profondeur ; enfin, de nombreuses recherches radiobiologiques intéressant les téguments ont été conduites chez le porc $[1,5,18,20,23]$.

\section{MATERIEL ET METHODES}

\section{II.1. Animaux}

A cause des différences morphologiques et physiologiques des tissus musculaires mâles et femelles, nous avons essayé, dans la mesure du possible, de ne travailler que sur des porcs femelles, les caractéristiques des castrés variant notamment suivant l'âge de la castration. Certains animaux ont reçu un traitement par voie générale : association d'anti-inflammatoires, d'anti-agrégants plaquettaires, de vasodilatateurs ou d'anticoagulants [9].

\section{II.2. Choix de la zone anatomique}

Les irradiations ont été appliquées sur la face externe de la cuisse droite à $3 \mathrm{~cm}$ en arrière du fémur, au niveau de la jonction du tiers proximal de l'os et du tiers moyen 
(fig.1). Les animaux sont anesthésiés à l'halothane dans un mélange gazeux $\mathrm{O}_{2}$ et $\mathrm{H}_{2} \mathrm{O}$.

La coupe de la cuisse réalisée perpendiculairement à l'axe du fémur (ligne $A B$ ) au point d'application de la source montre les différentes zones anatomiques exposées au rayonnement (fig. 2).

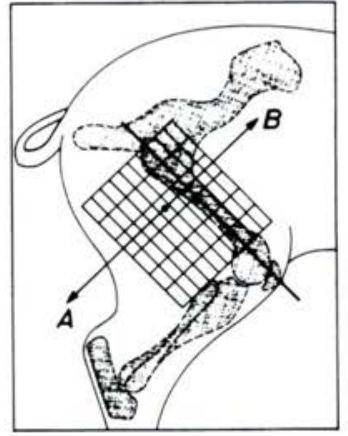

Fig.1. - Localisation des points de mesure thermographique selon une grille de 60 points.

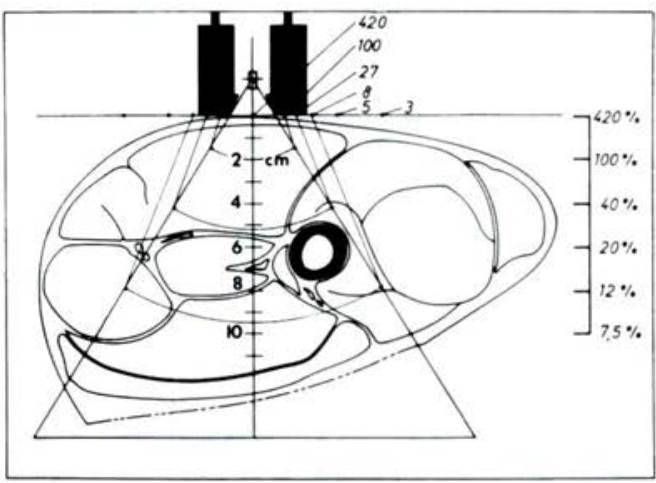

Fig. 2. - Courbes isodoses en profondeur et à la peau en pourcentage de la dose à $2 \mathrm{~cm}$.

\section{II.3. Protocole d'irradiation - Dosimétrie}

\section{II.3.1.}

L'irradiateur est un appareil de la CGR (Compagnie générale de radiologie) du type GR-50 utilisé en gammagraphie industrielle. Le déplacement de la source - ${ }^{192} \mathrm{Ir}$ de 200 à $50 \mathrm{Ci}$ - fixée à l'extrémité d'un câble est commandé électriquement. La partie libre du tube éjecteur est fixée dans un collimateur annulaire en plomb de $18 \mathrm{~mm}$ d'épaisseur (fig. 2).

- Conditions de l'irradiation

Deux types de collimateurs ont été utilisés :

- I'un (fig. 2) est constitué d'un anneau de plomb de $18 \mathrm{~mm}$ d'épaisseur dont l'extrémité appliquée sur l'animal est taillée en léger biseau et terminée par une fenêtre en plexiglass :

- dans l'autre (fig. 3), identique, on a ménagé une ouverture angulaire de $15 \mathrm{~mm}$ de hauteur et de 70 degrés d'angle au niveau de l'extrémité appliquée sur la peau pour étudier, sur une zone limitée, l'effet d'une source non collimatée ; une plaque de plexiglass est collée à l'extrémité du collimateur en débordant largement sur la zone exposée.

\section{II.3.2. Dosimétrie}

Le rendement en profondeur de la source d'iridium 192 placée dans le collimateur et appliquée sur un fantôme parallélépipédique de plexiglass est représenté sur la figure 5. Les mesures sont réalisées à l'aide d'une chambre d'ionisation de $0,6 \mathrm{ml}$ couplées à un dosimètre IONEX 2500/3. Pour des distances comprises entre 0,1 et $2 \mathrm{~cm}$ nous avons utilisé une chambre plate PTW de 0,03 $\mathrm{ml}$. 


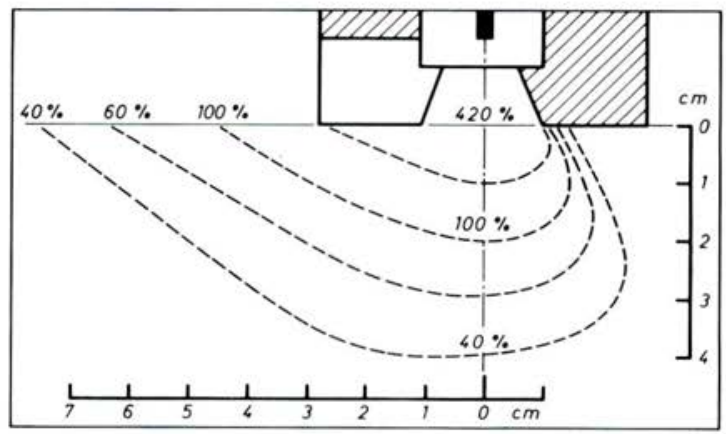

Fig. 3. - Courbes isodoses en profondeur en pourcentage de la dose à $2 \mathrm{~cm}$ avec le collimateur ouvert.

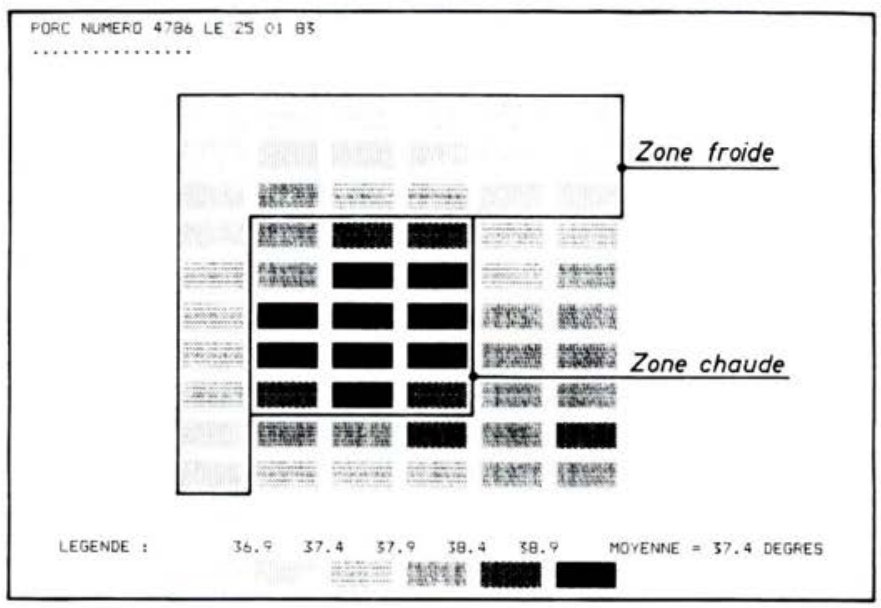

Fig. 4. - Délimitation des zones chaude et froide sur la grille de mesure.

La figure 2 représente les courbes isodoses au niveau de la peau et en profondeur pour trois pourcentages de la dose à $2 \mathrm{~cm}$ de profondeur: 100, 27 et 8 pour cent. La figure 3 représente, dans le cas du collimateur ouvert, quelques courbes isodoses en profondeur.

Nota bene: Les valeurs de doses d'exposition données dans la suite de ce texte correspondent à la valeur mesurée à $2 \mathrm{~cm}$ de profondeur. 


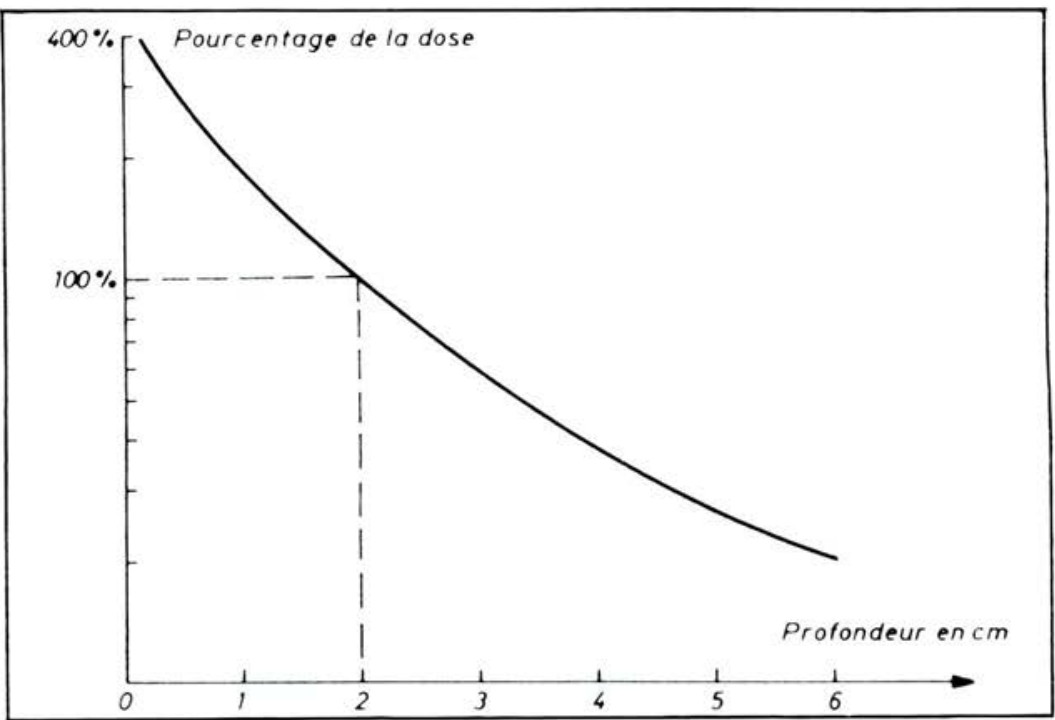

Fig. 5. - Courbe de rendement en profondeur de la source ${ }^{192} \mathrm{Ir}$. La valeur à $2 \mathrm{~cm}$ représente le $100 \%$.

\section{II.3.3. Doses utilisées}

Nous avons d'abord irradié les animaux à 84 Gy. En effet, au cours d'une expérience exploratoire, nous avons observé des restaurations spontanées systématiquement à 40 Gy (en 5 mois) et dans quelques cas - porcs mâles et âgés de 7-8 mois à 64 Gy (en 7 mois), alors que nous cherchions à obtenir une lésion durable. Dans un deuxième temps, nous avons choisi des doses plus faibles pour essayer divers traitements médicamenteux : 30,40 et 64 Gy.

Le débit de dose à $2 \mathrm{~cm}$ a varié de $6 \mathrm{~Gy} / \mathrm{min}$ pour une source de $200 \mathrm{Ci}$ à $1,5 \mathrm{~Gy} / \mathrm{min}$ au moment du changement de source, soit d'un facteur 4 : nous verrons plus loin que l'importance de ce facteur est susceptible de modifier la réponse à une irradiation locale, notamment dans le sens d'un retard dans la chronologie de l'évolution. C'est pourquoi nos expérimentations sont faites sur des séries d'animaux qui comportent toujours un témoin.

\section{II.4. Technique de mesure en thermographie microondes}

\subsubsection{Principe de fonctionnement [12, 14, 15, 21].}

Tout matériau porté à une température $\Theta$ émet un rayonnement électromagnétique d'origine thermique dont l'intensité et le spectre de fréquence dépendent de la tem. pérature, allant de l'infra-rouge aux ondes hertziennes. L'énergie émise dans le domaine de l'infra-rouge est $10^{6}$ fois plus forte que celle émise dans la bande des microondes. Si l'émission infra-rouge de longueur d'onde $9500 \mathrm{~nm}$ émise par le corps humain est arrêtée par une épaisseur de tissus de quelques micromètres, le rayonnement microondes $(\lambda=3-30 \mathrm{~cm})$ est beaucoup moins atténué. Selon la bande de fréquences utilisée, la profondeur explorée varie : dans un tissu à forte teneur en eau (muscle), les chiffres sont respectivement $0,4,2$ et $4 \mathrm{~cm}$ pour 9,3 et $1 \mathrm{GHz}$. En 
ce qui concerne la peau de la cuisse du porc, pour laquelle l'épaisseur de tissu adipeux sous-cutané est de $0,5-0,8 \mathrm{~cm}$, les profondeurs augmentent notablement : $0,6-0,7 ; 2,5-3$ et $4,5-5 \mathrm{~cm} \mathrm{[16]}$.

Les gradients de température sous-cutanée correspondant au plus à quelques degrés, les récepteurs doivent être sensibles à des variations de températưre de 0,1 degré : pour une bande passante de $1 \mathrm{GHz}$ la sensibilité doit être de l'ordre de $10^{-15} \mathrm{~W}$. Le thermographe microonde est donc constitué d'un récepteur à faible bruit, de grande sensibilité et d'un sonde (antenne) jouant le rôle d'interface avec les tissus.

\section{II.4.2.}

L'appareil utilisé est un TMO-3000 construit par Odam-Brucker (67160 - Wissembourg, France) en collaboration avec le Centre Hyperfréquences et semiconducteurs (LA CNRS 287) à l'Université des sciences et techniques de Lille. Sa fréquence de travail est de $3 \mathrm{GHz}$ et la surface active de la sonde de $2 \mathrm{~cm}^{2}(1 \times 2 \mathrm{~cm})$ (fig. 6).

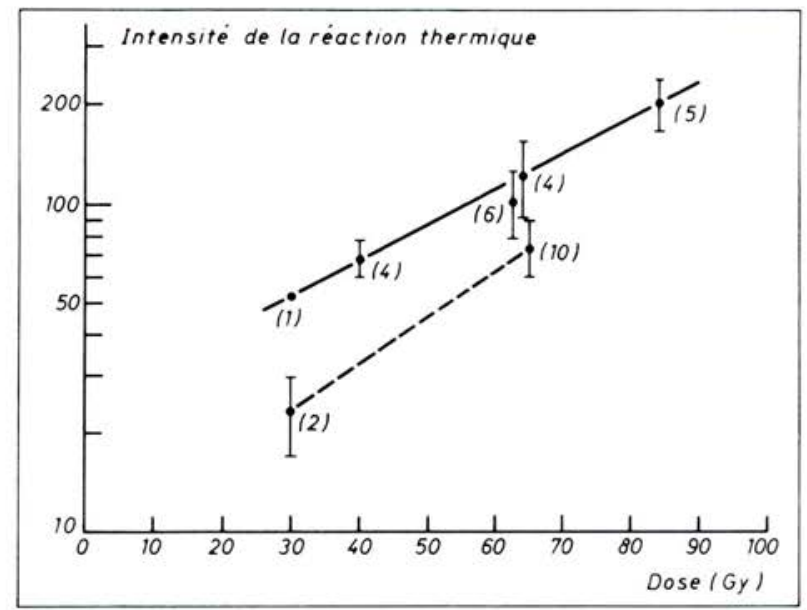

Fig. 6. - Relation entre la dose d'irradiation (à $2 \mathrm{~cm}$ ) et l'intensité de la réaction thermique : animaux normaux- - animaux traités

Les mesures ont toujours eu lieu le matin, 1 h 30 après la phase d'excitation due à la distribution du repas. L'animal est anesthésié $15 \mathrm{~min}$ avant la mesure et couché sur le côté gauche. Ce délai est indispensable pour que la température interne (et partant, superficielle) du sujet se stabilise après une chute de $0,5-0,7{ }^{\circ} \mathrm{C}$. II est également important, après avoir acclimaté les animaux à l'environnement du laboratoire (température, éclairement, rythme des repas), de les habituer aux manipulations dues aux anesthésies quotidiennes qui provoquent, au début; des réactions de défense retentissant sur la température interne.

Soixante points de mesure sont réalisés quotidiennement sur chaque animal quelques jours auparavant et pendant au moins 20 jours après irradiation, selon les grilles représentées aux figures 1 et 7 : il s'agit d'un fin grillage de matière plastique dans lequel ont été découpés 60 trous rectangulaires de $1 \times 2 \mathrm{~cm}$. La surface cutanée mesurée est égale à $196 \mathrm{~cm}^{2}(13,5 \times 14,5 \mathrm{~cm})$. La sonde est placée au contact de la peau à travers chaque fenêtre et, après un court temps de mise en équilibre ( 3 à $5 \mathrm{~s})$, la température mesurée est enregistrée et mise en mémoire sur un calculateur APPLE II. Selon le constructeur, la valeur de la température est donnée à $\pm 0,15^{\circ} \mathrm{C}$. 

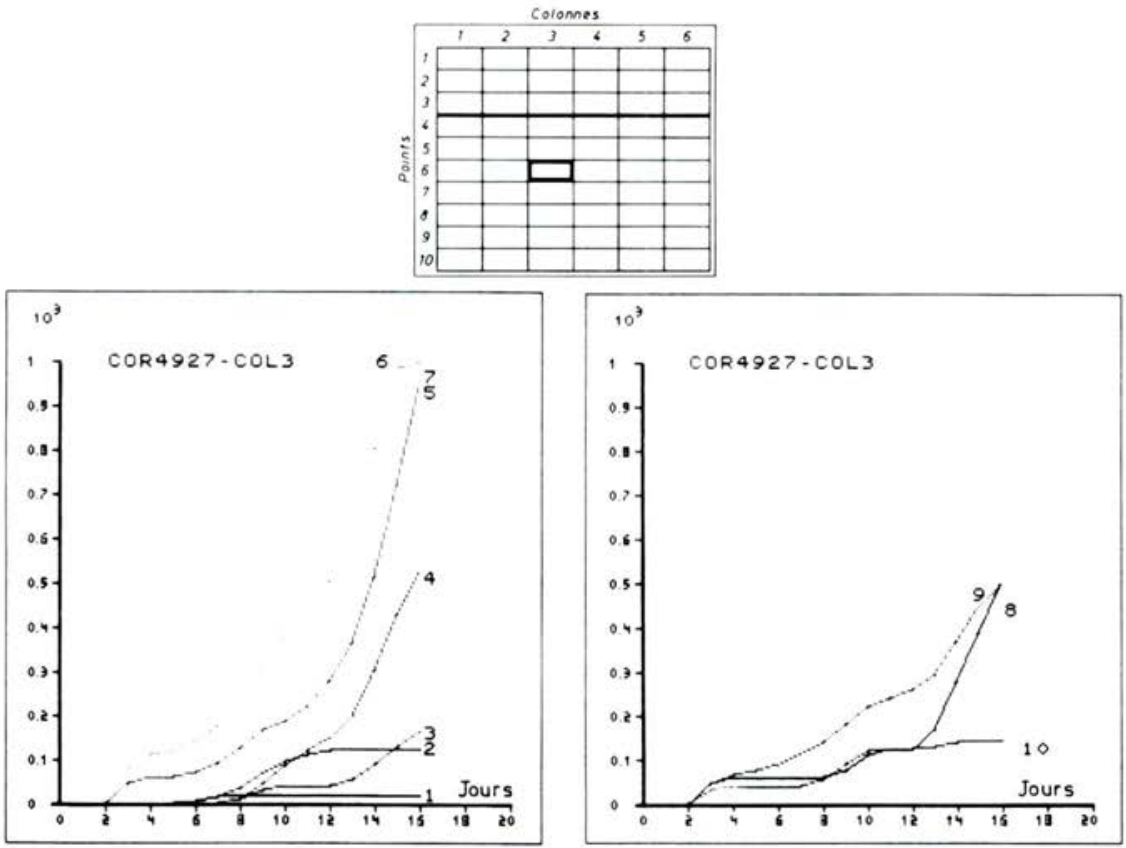

Fig. 7. - Collimateur fermé. Intensité de la réaction thermique (cumulée sur 16 jours) pour les 10 points de la colonne 3 . Le point d'application de la source est en $6-3$.

Dans le cas d'une irradiation avec le collimateur ouvert, les mesures sont réalisées selon une grille de 91 points $(13$ lignes et 7 colonnes $-17,5 \times 19 \mathrm{~cm}$, soit $332 \mathrm{~cm}^{2}$ ) (fig. 8).

\section{II.5. Mise en forme et traitement des données}

\section{II.5.1. Entrée des données}

Le signal (tension continue) issu du TMO.3000 est envoyé dans un microordinateur APPLE II où il fait l'objet d'une conversion analogique - digitale avant d'être pris en charge par un programme de saisie de données. Au début de chaque ligne de 6 mesures, la température interne de l'animal déterminée par une sonde rectale est automatiquement mémorisée : on obtient donc un tableau de 10 lignes de 7 valeurs (ou 13 lignes de 8 valeurs) qui s'inscrit sur l'écran.

\subsubsection{Aspect semi-quantitatif}

Deux types de représentation sont proposés :

- une représentation de la grille de mesure par des pavés rectangulaires correspondant à la surface utile de la sonde : les 6 teintes de gris utilisées correspondent à un histogramme en 6 classes des températures mesurées (fig. 9) ;

- une transformation de la grille de $6 \times 10$ en $19 \times 21$ valeurs par interpolation linéaire, perpendiculaire et diagonale, permet de faire imprimer une image de 399 points, avec les mêmes 6 teintes de gris (fig. 10) ; 

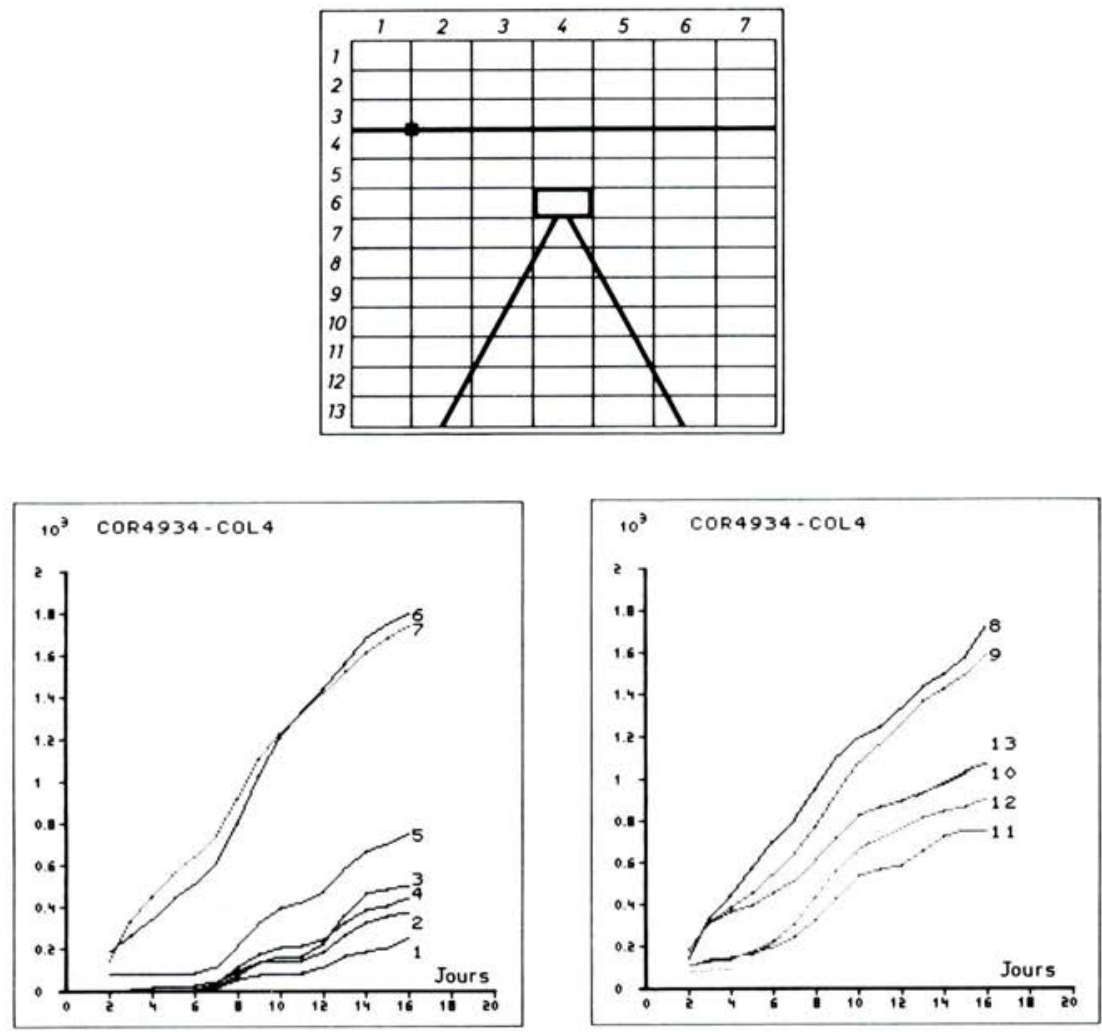

Fig. 8. - Collimateur ouvert. Intensité de la réaction thermique (cumulée sur 16 jours) pour les 13 points de la colonne 4 . Le point d'application de la source est en $6-4$.

\subsubsection{Aspect quantitatif}

Le programme de calcul s'efforce de condenser l'information contenue dans une grille de mesure en un index significatif de l'intensité de la réaction thermique au niveau de la lésion. La méthode la plus satisfaisante consiste à définir une zone de 25 points répartis à la périphérie de la grille dite "zone froide " - et une zone de 15 points centrée sur la lésion dite "zone chaude" et à les comparer au cours du temps avant et après irradiation (fig. 4). La zone chaude peut être limitée au point d'application de la source ou aux 8 points qui l'entourent.

La zone placée en bas à droite de la grille correspondant à l'articulation fémorotibiale n'est pas stable thermiquement.

\section{RESULTATS}

\section{III.1. Aspects cliniques - Chronologie de l'apparition des lésions}

En prenant comme modèle une irradiation à $84 \mathrm{~Gy}$, on observera successivement les phases suivantes: 


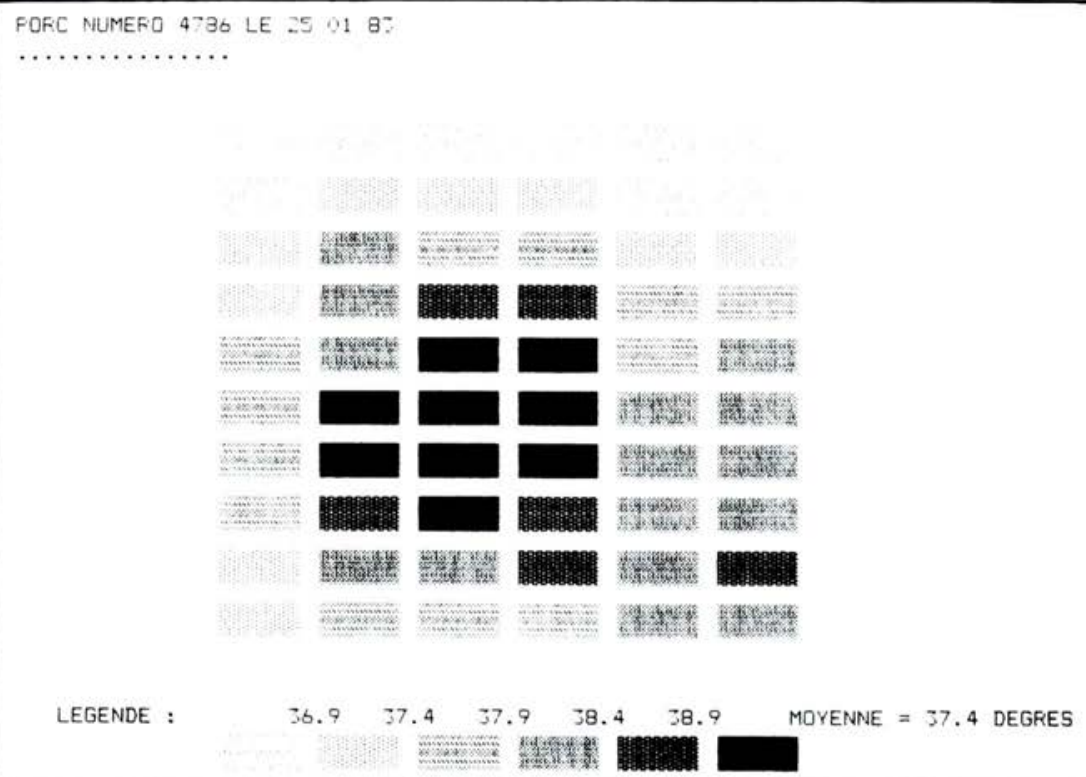

Fig. 9. - Résultats bruts d'une mesure thermographique représentés selon une échelle de 6 valeurs de gris.

- Assez fréquemment, un érythème est visible immédiatement après la fin de l'irradiation, qui s'affaiblit ou - le plus souvent- disparaît dans les heures qui suivent.

- Un érythème précoce apparaît après $18-24 \mathrm{~h}$ et dure 48 à $72 \mathrm{~h}$ (planche 1, II-III).

- Une phase de latence lui succède, au cours de laquelle la réaction érythémateuse s'atténue et disparaît parfois complètement (planche 1, IV) ; elle prend place entre le $4^{e}$ et le $5^{e}$ jour en moyenne. Une réaction oedemateuse, simulant une large papule de $2 \mathrm{~cm}$ de diamètre, accompagne fréquemment les trois premières phases.

- Une phase de thrombose où la lésion devient lilas puis violet de plus en plus foncé tandis qu'elle s'épaissit, entre le $5^{e}$ et le $9^{e}$ jour.

- Une phase d'épidermolyse suivie de l'installation d'une escarre entre le $10^{\circ}$ et le $13^{e}$ jour.

- Puis, la lésion s'étendant en profondeur, un ulcère se creuse sous la croûte qu'un sillon va bientôt, en s'élargissant, détacher des tissus environnants (planche $1, \mathrm{~V}, \mathrm{VI}, \mathrm{VII}$ ).

- Une sorte de "bouchon" de tissu nécrosé s'individualise alors, avant d'être éliminé : l'ulcère profond de 2 à $3 \mathrm{~cm}$ ainsi installé va évoluer vers une fistule en s'enfouissant progressivement tandis que les bords de la plaie se referment en laissant un pertuis de quelques millimètres de dia- 
F. DABURON, J.L. LEFAIX, J. REMY, G. FAYART, Y. TRICAUD
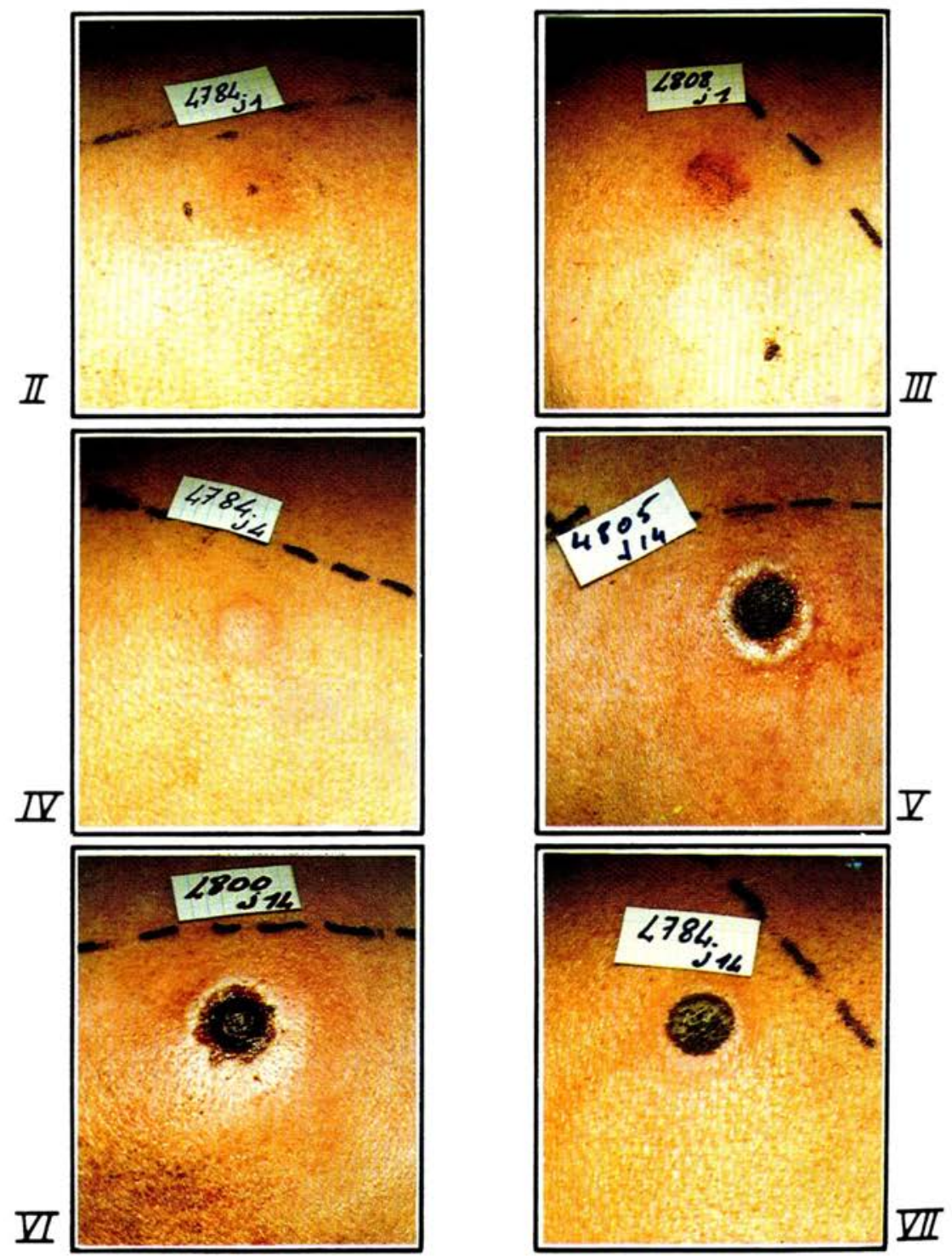

Planche 1 
mètre. A 84 Gy, il n'y a pas de cicatrisation spontanée et l'évolution de la lésion s'arrête à ce stade, tout au moins pendant toute la durée de nos observations (jusqu'à 8 mois).

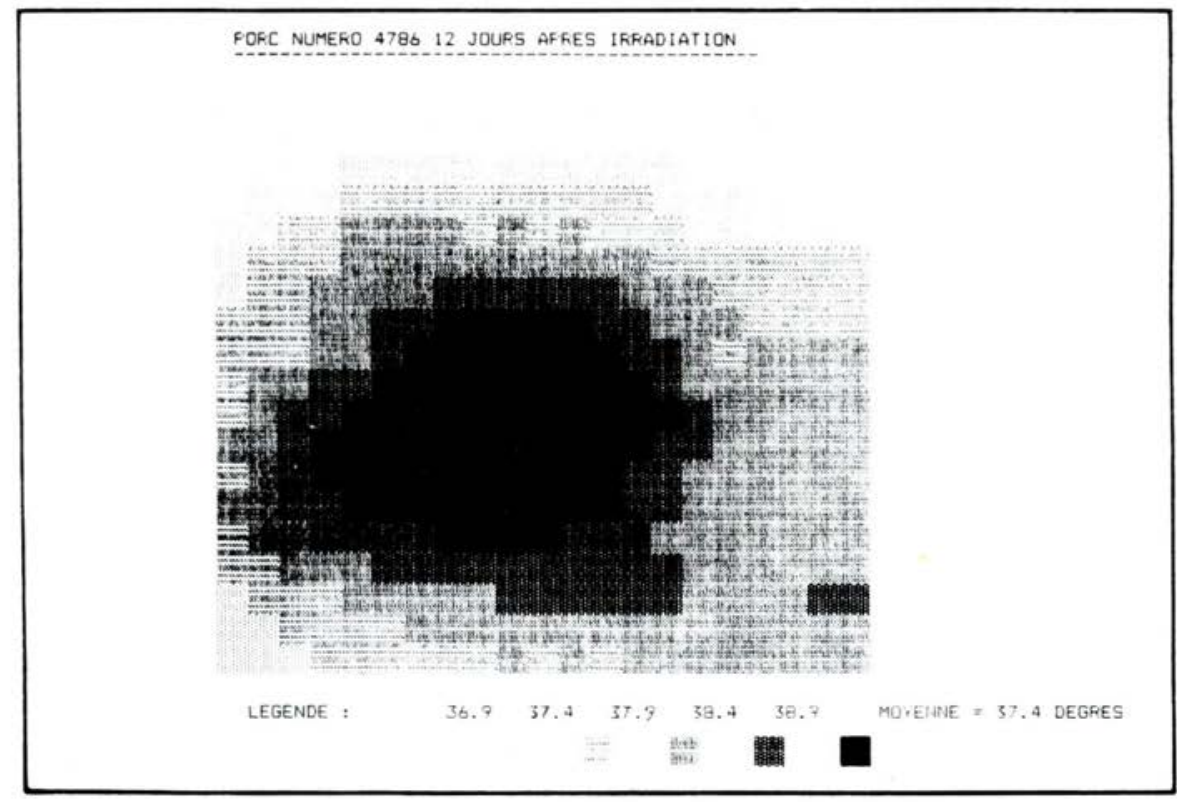

Fig. 10. - Transformation de la grille de mesure en image par interpolation selon les axes verticaux et horizontaux.

Pour 64 Gy assez fréquemment et, dans tous les cas, pour 40 Gy, la cicatrisation s'opère très lentement et après 5 mois environ la plaie s'est refermée complètement, il ne reste qu'une cicatrice fibreuse sous-cutanée de 4 à $6 \mathrm{~cm}$ de largeur et qui s'enfonce de 3 à $5 \mathrm{~cm}$ dans le tissu musculaire.

La figure 11 représente une évolution moyenne, pour 4 valeurs de doses, de l'aspect anatomo-clinique des lésions : 155 Gy ( 1 animal), 84 Gy (14 animaux), 64 Gy (12 animaux), 40 Gy (6 animaux). On voit très nettement que l'augmentation de la dose entraîne une accélération dans la chronologie de l'apparition des lésions.

Enfin, il ne semble pas, dans cette localisation de la radiolésion et compte tenu de la séméiologie relativement fruste chez ces animaux, que la douleur - atroce dans de nombreux cas décrits chez l'homme - ait joué un rôle important dans les modifications du comportement des sujets.

\section{III.2. Aspects cliniques et thermographie microondes qualitative}

La planche 2 représente les images thermographiques des lésions figurées sur la planche 1 ; la thermographie I est une image témoin. Dans de nombreux cas on a, pour le même aspect anatomo-clinique, des images thermographiques très différentes (planche 2, II, III, et IV). On voit, chez 
F. DABURON, J.L. LEFAIX, J. REMY, G. FAYART, Y, TRICAUD
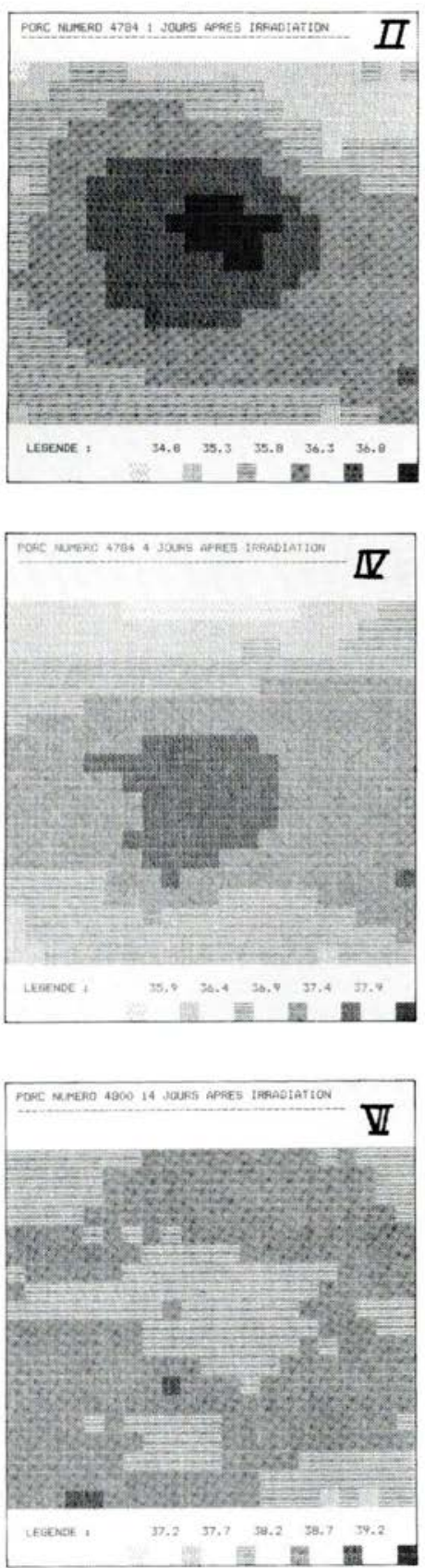
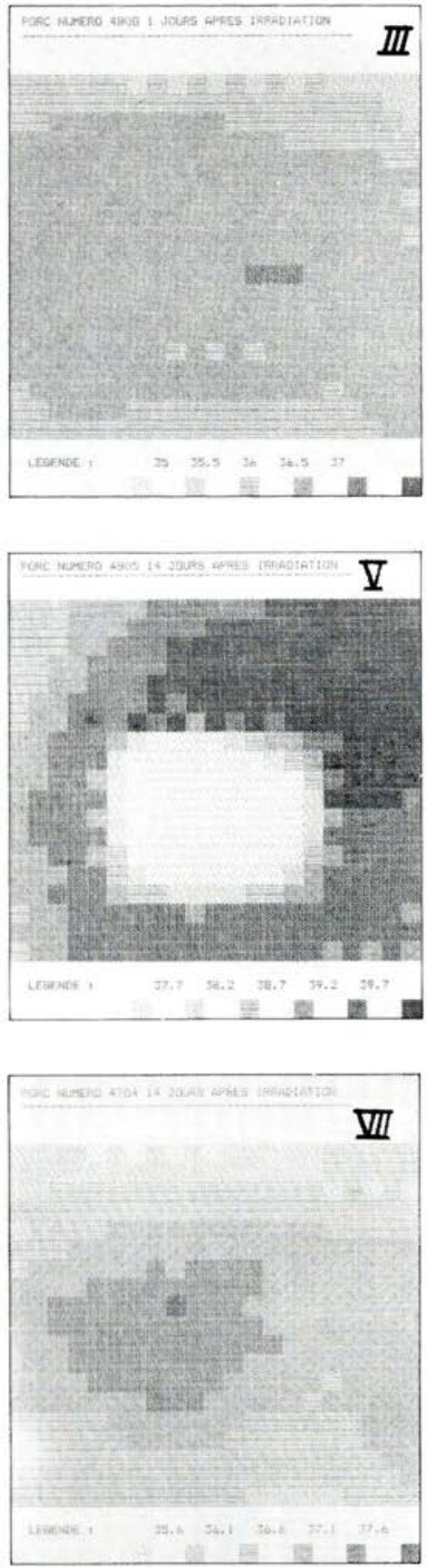

Planche 2

RADIOPROTECTION 
certains sujets, que d'un jour à l'autre l'image thermographique évolue très rapidement avec apparition de zones hypo ou hyperthermiques en $24 \mathrm{~h}$. - L'apparition d'une zone froide au niveau de la lésion signe l'installation d'une nécrose importante et profonde; toutefois, au niveau de la périphérie, apparaît souvent une réactivation thermique et il semble que cette zone soit le siège de phénomènes passagers de vasoconstriction et de vasodilation (planche 2, V, VI et VII).

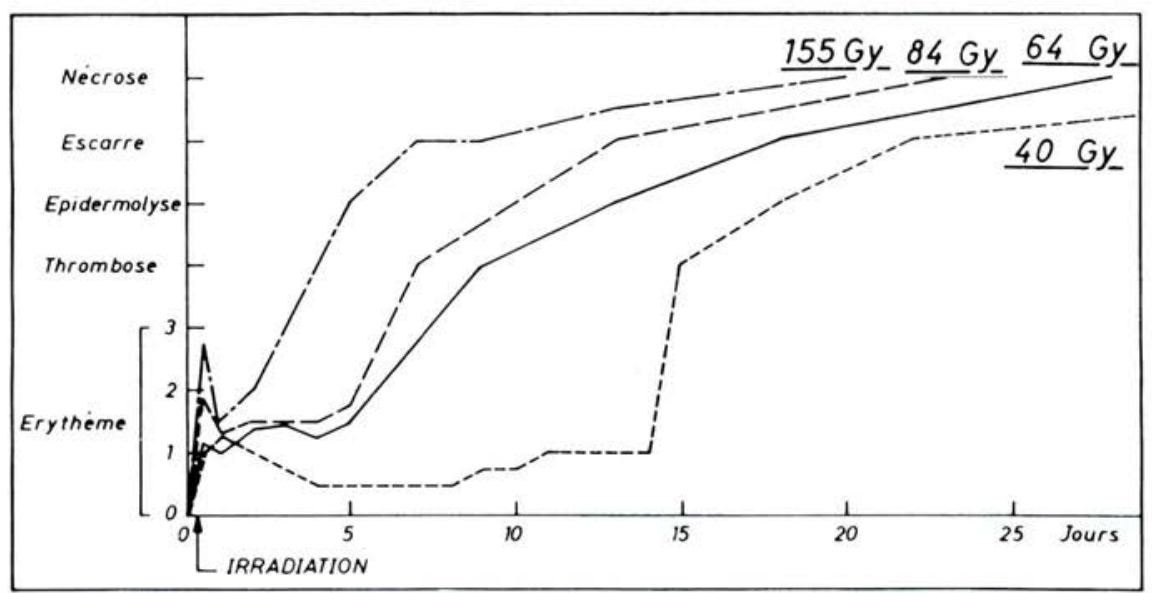

Fig. 11. - Chronologie de l'évolution des lésions en fonction de la dose.

\section{III.3. Essai de quantification de la réaction thermique - applications}

\section{III.3.1. Loi dose-effet}

Nous avons vu le principe du calcul au paragraphe 11.5.3. : on étudie quotidiennement la différence entre la température moyenne de la zone "chaude" (15 points centrés sur la lésion) et la température moyenne de la zone "froide" périphérique (25 points).

Le tableau II donne, pour 4 valeurs de dose, la moyenne et l'écart type de ces différences cumulées - en $1 / 10$ de degré - sur 20 jours pour chaque sujet, et soustraite de la valeur témoin (valeur moyenne des différences mesurées sur 30 porcs témoins, multipliée par $20 \mathrm{j}$ ). Si, pour des doses fortes ( 84 et $64 \mathrm{~Gy}$ ), le cumul des différences s'aligne selon une droite de régression significative dès le $10^{e}$ jour, il faut attendre le $20^{e}$ jour pour les doses faibles (30 et $40 \mathrm{~Gy}$ ) où la réaction thermique est plus faible.

TABLEAU ।

\begin{tabular}{|l|c|c|c|}
\hline Dose (Gy) & Nb d'animaux & Différences cumulées & $\sigma / \sqrt{n}$ \\
\hline 84 & 5 & 200 & 36 \\
64 & $4(6)^{*}$ & $119(100)^{*}$ & $30(22)^{*}$ \\
40 & 4 & 68 & 8 \\
30 & 1 & 52 & -14 \\
64 traités & 10 & 73 & 6 \\
30 traités & 2 & 23 & \\
\hline
\end{tabular}

* + 2 mâles castrés assez tardivement (4 mois) (cf II-1). 
La figure 6 illustre la proportionnalité entre la dose et le logarithme des moyennes des différences entre la zone chaude irradiée et la zone froide témoin (somme des différences cumulées sur 20 jours).

\section{III.3.2. Corrélation entre la dose, les données cliniques et thermographiques}

Le tableau III représente la matrice de corrélation entre 8 paramètres expérimentaux mesurés sur 43 animaux après une analyse en composantes principales [8]. Pour simplifier les calculs, chaque paramètre a été divisé en 3 classes d'effectifs à peu près égaux (tableau II).

\section{TABLEAU ॥}

(Le nombre de sujets est entre parenthèses)

\begin{tabular}{|c|c|c|c|c|c|c|}
\hline \multirow[b]{2}{*}{ Dose en Gy } & \multicolumn{2}{|c|}{ Classe 1} & \multicolumn{2}{|c|}{ Classe 2} & \multicolumn{2}{|c|}{ Classe 3} \\
\hline & $30-40$ & (13) & 64 & (21) & 84 & ( 9 ) \\
\hline Débit de dose en Gy/min & $0,66-1,35$ & $(16)$ & $2,4-3,6$ & $(14)$ & $4,5-5,5$ & (13) \\
\hline \multicolumn{7}{|c|}{ Dates d'apparition des lésions ( $\mathrm{JO}=$ jour de l'irradiation) : } \\
\hline Thrombose & J5-J6 & (14) & J7-J8 & $(14)$ & J9 & (15) \\
\hline Epidermolyse & $\mathrm{J} 7-\mathrm{J} 8$ & (11) & J9-J11 & $(16)$ & J12 & (16) \\
\hline Escarre & J9-J11 & $(16)$ & J12-J15 & $(14)$ & $\mathrm{J} 20$ & (13) \\
\hline Nécrose & J13-J19 & (17) & $\mathrm{J} 2 \mathrm{O}-\mathrm{J} 24$ & (13) & $\mathrm{J} 27$ & (13) \\
\hline $\begin{array}{l}\text { Somme des } \\
\text { différences cumulées }\end{array}$ & $0-30$ & $(11)$ & $40-74$ & (15) & 92 & (17) \\
\hline $\begin{array}{cc}\text { Traitement } & O(22) \\
\text { (Daburon 1985) } & \end{array}$ & $\begin{array}{r}\text { Anticoagular } \\
\text { vasodilatate }\end{array}$ & $\begin{array}{l}\text { int }(6) \\
\text { eeurs }\end{array}$ & & $\begin{array}{l}\text { Anti-in } \\
\text { Anti-ag }\end{array}$ & $\begin{array}{l}\text { mmatoire } \\
\text { gants (15 }\end{array}$ & \\
\hline
\end{tabular}

TABLEAU III

Matrice de corrélation

\begin{tabular}{|lrrrrrrrr|}
\hline Dose & 1000 & & & & & & & \\
Débit & 69 & 1000 & & & & & & \\
Thrombose & -610 & -70 & 1000 & & & & & \\
Epidermolyse & -596 & 51 & 870 & 1000 & & & & \\
Escarre & -692 & -42 & 845 & 837 & 1000 & & & \\
Nécrose & -765 & 59 & 799 & 760 & 846 & 1000 & \\
Somme & 477 & 337 & -399 & -289 & -271 & -333 & 1000 & \\
Traitement & -347 & -46 & 63 & 63 & 234 & 225 & -338 & 1000 \\
\hline
\end{tabular}

Le seuil de significativité est de 0,39 à $1 \%$ et 0,30 à $5 \%$. On voit des corrélations très significatives entre la dose et le délai d'apparition des radiolésions (par exemple -0,61 pour l'apparition de la thrombose) : il n'y a pas de corrélation avec le débit de dose. Par contre, celui-ci est corrélé, comme la dose, avec la somme des différences de températures (zone chaude irradiée - zone froide témoin) c'est-à-dire avec la réaction thermique locale. 
Les traitements diminuent la réaction thermique $(r=-0,338)$ et agissent comme une diminution de la dose d'exposition ( $r=-0,347)$. Les effets sur le délai d'apparition des lésions ne sont pas significatifs avec cette technique de classification, mais ils sont très nets dans chaque groupe entre témoins et traités [9].

\section{III.3.3. Remarques sur les mesures thermographiques microondes pour} notre experimentation chez le porc

III.3.3.1. Influence de la température interne sur les mesures en thermographie microondes

Une étude réalisée sur 86 grilles de mesures obtenues sur 20 animaux témoins, c'est-à-dire pendant les jours qui précédaient l'irradiation, a permis d'établir une loi linéaire simple de correction $(r=0,70, t=9)$.

$$
\text { T } \mu \text { o corrigée }=26,6+(T \mu \text { o mesurée }-26,6) \times \frac{T \text { interne standard }}{T \text { interne mesurée }}
$$

$T \mu \mathrm{O}:$ température microondes, température interne standard : $37,2{ }^{\circ} \mathrm{C} \pm 1{ }^{\circ} \mathrm{C}$ (moyenne obtenue sur 86 mesures sur 20 animaux).

Cette correction a été appliquée aux résultats pour pouvoir comparer les images d'un jour à l'autre, alors que les températures internes peuvent varier de $\pm 2{ }^{\circ} \mathrm{C}$; elle est évidemment inutile pour les calculs d'index où l'on ne considère que les différences entre les températures moyennes de la zone chaude et les températures moyennes de la zone froide.

III.3.3.2. Relation entre les mesures thermographiques et les mesures en profondeur dans les tissus à l'aide d'un thermocouple

Cette étude a été entreprise sur six animaux selon le protocole suivant : les animaux, préalablement anesthésiés, demeuraient couchés sur le côté droit pendant un quart d'heure environ pour que la température rectale soit à peu près stable ; une mesure microondes était réalisée sur la cuisse gauche en un point symétrique du point d'application de la source sur la cuisse droite; puis, en ce point, la peau et l'aponévrose du biceps fémoral étaient percées à l'aide d'un petit trocard pour laisser passer le thermocouple constitué d'une tige métallique de $120 \mathrm{~mm}$ de long et de $1 \mathrm{~mm}$ de diamètre à l'extrémité de laquelle se trouve le couple $\mathrm{Ni}-\mathrm{Cr} / \mathrm{Ni}$ allié (fem. $41 \mu \mathrm{V}$ par degré). La tige était enfoncée perpendiculairement à la surface de la peau, et retirée progressivement tandis que la température correspondante à chaque profondeur était notée.

\section{RESULTATS}

De 10 à environ $2,5 \mathrm{~cm}$ de profondeur, la température est égale, à quelque $1 / 10$ de degré près, à la température rectale, puis chute rapidement pour atteindre, à $2 \mathrm{~mm}$ de profondeur - au contact de l'aponévrose du biceps fémoral - une valeur inférieure de $2,5^{\circ} \mathrm{C}$ à la température centrale (température rectale - température superficielle $=2,5 \pm 0,5 ;$ valeurs extrêmes $1,8-3,1$ ).

La mesure microondes donne pour 6 animaux $36,5 \pm 0,50{ }^{\circ} \mathrm{C}$ pour une température rectale de $38,4 \pm 0,20^{\circ} \mathrm{C}$. La profondeur où la température lue sur le thermocouple correspond à celle affichée sur le thermographe microonde est de $0,80 \pm 0,3 \mathrm{~cm}$ (valeurs extrêmes $0,35-1,5$ ). 


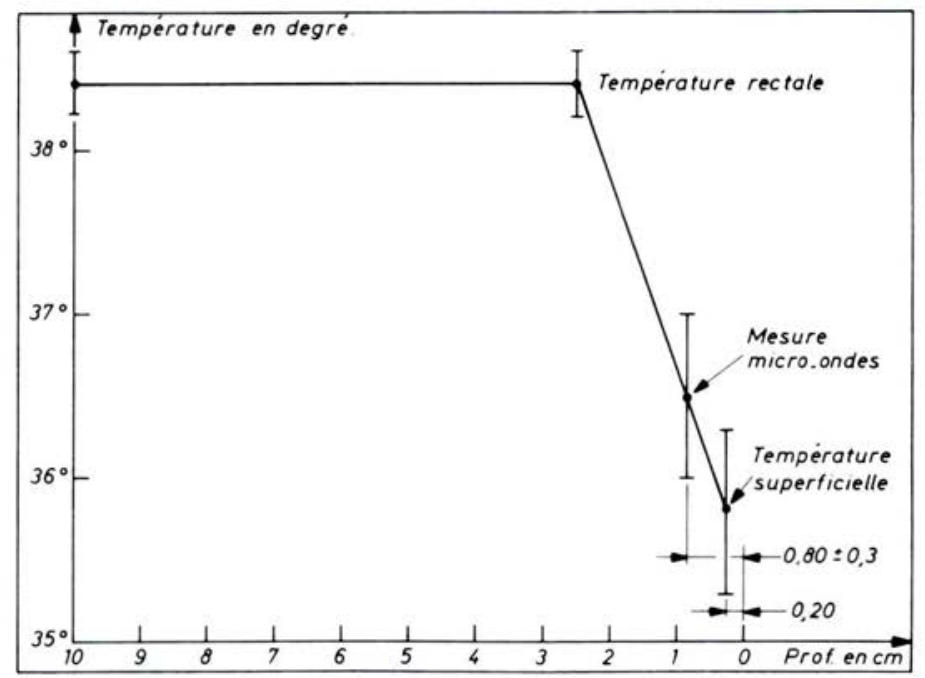

Fig. 12. - Evolution moyenne de la température en fonction de la profondeur dans la cuisse d'un porc témoin ; mesure par thermocouple.

La figure 12 schématise l'évolution moyenne de la température en fonction de la profondeur de la cuisse d'un porc témoin. II est important de noter que la température microondes mesurée en quelques points de la zone chaude de la grille, au moment de certaines poussées inflammatoires, a été supérieure de 0,5 à $1^{\circ} \mathrm{C}$ à la température rectale; ce fait plaide en faveur de l'hypothèse de l'existence de 2 composantes de la réaction thermique locale après irradiation : augmentation de la circulation locale et augmentation du métabolisme tissulaire (surtout musculaire).

\section{III.4. Essais de diagnostic de l'étendue de la zone irradiée par les mesures thermographiques microondes}

A l'aide d'un analyseur d'image Pericolor (Numelec), nous avons sommé successivement chaque grille de mesures journalières (grilles $10 \times 6$ et $13 \times 7$ ) après soustraction, pour chacune, d'une grille témoin. Cette méthode fait apparaître sur l'écran une zone d'hyperthermie permanente qui se détache bien de la zone froide où les variations de température sont plus aléatoires.

Si l'on étudie l'évolution dans le temps de chaque point de la grille on obtient des courbes analogues à celles représentées fig 7 et 8 . Fig. 7 : Porc 4927 - 64 Gy - collimateur annulaire fermé. La zone réactive s'étend sur 3 points $(5,6,7)$ des colonnes 3 et 4 ; les courbes correspondant à ces points se détachent nettement de celles représentant les points environnants. Fig. 8 : Porc 4934 - 30 Gy - collimateur annulaire ouvert. La zone centrale qui va desquamer représente les points $6,7,8$ et 9 de la colonne 4 et se détache nettement des autres points : elle correspond à la zone 
ayant reçu plus de 20 Gy à la peau. La zone réactive s'étend sur les colonnes 3,4 et 5 , à partir de la ligne 5 , jusqu'à la ligne 13 .

\section{DISCUSSION}

Les résultats des mesures thermographiques microondes peuvent être exploités selon deux aspects plus ou moins intriqués :

- aspect diagnostique au niveau de la zone irradiée : quelle est l'étendue des radiolésions, leur intensité et quel est le pronostic?

- aspect physiopathologique de l'évolution de la réaction thermique locale en liaison avec l'aspect clinique ; influence d'un traitement par voie générale.

L'aspect diagnostique est simplifié dans notre modèle où l'emploi d'un collimateur permet de délimiter de façon très tranchée, en surface comme en profondeur, les zones irradiées des zones saines. Nous avons vu que l'intensité de la réaction thermique locale était proportionnelle à la dose d'exposition : ceci est un élément important du diagnostic mais doit être corrigé par l'influence du débit.

Diverses difficultés s'ajoutent aux considérations précédentes dans l'optique d'une évaluation dosimétrique : la variabilité individuelle, l'âge, le sexe des animaux, ce qui nous a conduits à étudier les expérimentations par lots, avec toujours un ou deux témoins; le défaut d'homogénéité thermique de la zone irradiée ; l'état physiopathologique du sujet et, en particulier, sa température centrale ; nous avons vu, toutefois, au paragraphe précédent (III.4) que l'analyse point par point de l'évolution thermographique locale permettait assez rapidement (avant 10 jours) de distinguer la zone irradiée - chaude - des zones périphériques plus froides.

Les mesures thermographiques microondes apportent des renseignements très précieux sur l'évolution de la réaction thermique locale en liaison avec l'aspect anatomo-clinique. L'érythème des premiers jours disparaît plus ou moins longtemps (de 1 à 10 jours) selon la valeur de la dose, avant l'apparition de la thrombose et de l'épidermolyse. Cependant, des périodes d'hyperthermie profonde sont mesurables sans aucun retentissement superficiel (planche 1, IV). L'apparition d'une zone d'hypothermie centrée sur le point d'application de la source d'irradiation sur la peau, puis débordant sur la périphérie, signe l'apparition d'une nécrose qui, dans notre modèle, est la règle après 84 et 64 Gy mais non après 40 et 30 Gy ; chez certains animaux traités après avoir reçu 64 Gy, cette zone "froide" n'apparaît pas.

Enfin, cette mesure globale permet d'apprécier l'effet de certains traitements administrés par voie générale sur la réaction thermique locale. Nous avons vu, d'autre part [9], que cette moindre réactivité thermique était liée à un retard dans l'évolution chronologique des lésions et à une limitation de l'extension de la fibrose cicatricielle (quand la cicatrisation est possible). 


\section{v. CONCLUSION}

Les mesures thermographiques infrarouges sont utilisées depuis longtemps pour le diagnostic des irradiations aiguës localisées chez I'homme [22].

Une amélioration, portant sur l'extension du champ de mesure en profondeur, a été proposée pour l'utilisation des sondes thermographiques microondes [16]. Nous avons étudié les possibilités de cette nouvelle technique vis-à-vis du diagnostic de l'étendue et de l'intensité des radiolésions locales obtenues chez le porc après des irradiations comprises entre 30 et 84 Gy (à $2 \mathrm{~cm}$ de profondeur). Les résultats sont intéressants, mais il est certain que l'emploi de sondes travaillant à des longueurs d'onde différentes et donc mesurant à des profondeurs différentes, apporterait des renseignements beaucoup plus précis sur la répartition des atteintes profondes et superficielles, en délimitant un certain "volume thermique". Nous poursuivons actuellement des recherches sur le sujet en collaboration avec le Centre Hyperfréquences et semiconducteurs de l'Université de Lille I (professeur Y. Leroy).

Mais, déjà, avec notre sonde travaillant à $3 \mathrm{GHz}$ qui prend en compte les contributions thermiques des différentes couches sous-épidermiques jusqu'à 2 ou $3 \mathrm{~cm}$ de profondeur, nous avons pu mettre en évidence l'évolution de la réaction thermique locale après une irradiation aiguë et les modifications que lui apportent certains facteurs (dose, débit, traitement).

\section{BIBLIOGRAPHIE}

[1] ARCHAMBEAU J.O., MATHIEU G.R., BRENNEIS H.J., THOMPSON K.R., FAIRCHILD R.G. The response of the skin of swine to increasing single exposures of 250-kVp X-rays. Rad. Res., 1968, 36, 299-326.

[2] ARCHAMBEAU J.O., MATHIEU G.R., BRENNEIS H.J., THOMPSON K.R. The response of the skin of swine to increasing multiple exposures of $X$-Ray (250 $k V p)$. Rad. Res., 1969, 37, 141-160.

[3] ARCHAMBEAU J.O. The response of the skin of swine to increasing absorbed doses of the ${ }^{10} \mathrm{~B}(\mathrm{n}, \alpha)^{7} \mathrm{Li}$ reaction : histologic and cytologic changes. Rad. Res., $1971,45,137-144$.

[4] ARCHAMBEAU J.O., FAIRCHILD R.G., BRENNEIS H.J. The response of the skin of swine to increasing absorbed doses of radiation from a thermal neutron beam, a degraded fission neutron beam, and the ${ }^{10} \mathrm{~B}(\mathrm{n}, \alpha)^{7} \mathrm{Li}$ reaction. Rad. Res., 1971 , $45,145-165$.

[5] ARCHAMBEAU J.O., BENNETT G.W., ABATA J.J., BRENNEIS H.J. Response of swine skin to acute single exposures of $X$-ray: quantification of the epidermal cell changes. Rad. Res., 1979, 79, 298-337.

[6] ASSOCIAZONE ITALIANA DI RADIOPROTEZIONE MEDICA. II sistema tegumentaria et le radiazioni ionizzanti. 3 Congresso Nazionale, Saint Vincent, 13-15 Sept.1981. (STRAMBI E., Ed.). Rome : Arte Grafiche. F. Santarelli, 1981.

[7] BARRETT A.H., MYERS P.C. Microwave thermography, a method of detecting subsurface thermal patterns thermography. In : Proceedings of the First European Congress, Amsterdam, 1974. Bibl. Radiol N.6, 1975, 45-56. 
[8] CAILLEZ F., PAGES J.P. Introduction à l'analyse des données. Paris: SMASH, 1976.

[9] DABURON F., LEFAIX J.L., REMY J., MARTIN M., BOLNOT D., GUIBAUD J., VEROLA O. Evolution des lésions après une irradiation aiguë localisée chez le porc. Essais de traitement médical et chirurgical. Rapport DPS 84/05 1985.

[10] EDRICH J., HARDEE P.C. Microwave radiometric detection and location of breast cancer. Proc. IEEE, 1974, 62, 1391-1392.

[11] ENANDER B., LARSON G. Microwave radiometric measurements of the temperature inside the body. Electronics letters, 1974, 10, 817.

[12] GAUTHERIE M., GUERQUIN-KERN J.L., KOTEWICZ A. Microwave thermal imaging : technical advances and clinical findings. Third International congress of thermology, Bath (England) 29 mars - 2 avril 1982.

[13] GONGORA R. Communication personnelle.

[14] HUBNER K.F., FRY S.A. Radiation accidents : Acute local irradiation. In : The Medical basis for radiation accident preparedness, Proceedings of the REAC/TS international conference, Oak Ridge, Tennessee, USA October 18-20, 1979 New York Elsevier/North-Holland. 1980, p 167-279.

[15] LEFAIX J.L., DABURON F., VEROLA O., BROCHERIOU Y., TRICAUD Y., HAAG J. Analyse anatomo-phathologique du processus lésione! après irradiation aiguë localisée chez le porc. Rapport DPS-84/01 SPE, 1984.

[16] LEROY Y. Microwave thermography of biomedical applications. Workshop on medical applications of microwaves. 12 th European Microwave Conference, Helsinki (Finlande) Sept 1982.

[17] MAMOUNI A., NGUYEN D.D., ROBILLARD M., CHIVE M., LEROY Y. Thermographie microondes. L'onde Electrique, 1980, 60 (12) 30-36.

[18] MORRISON R.A., ZELLMER D.L., RIDENHOUR R.L., DEAN R.D. Fractionnation and dose-rate effects on the acute reaction of pig skin to low-intensity gamma rays. Int. J. Radiat. Oncol. Biol. Phys., 1980, 6, 1679-1684.

[19] MOUSTAFA H.F., HOPEWELL J.W. Blood flow clearance changes in pig skin after single doses of X-rays. Br. J. Radiol., 1979, 52, 138-144.

[20] RENNER K., RENNER H. Experimentelle Untersuchungen über das Auftriten und den Verlauf das Früherythems an der Schweinhaut. Strahlentherapie, $1971,142,219-226$.

[21] ROBILLARD M. Contribution à l'étude des sondes et à la reconnaissance d'objet thermique par thermographie microondes. Thèse $3^{e}$ cycle, Université des Sciences et Techniques de Lille, 1981.

[22] STRAMBI E. Problemi di sorveglianza medica nelle radiodermiti acute e croniche. In : Il sistema tegumentario e le radiazioni ionizzanti. Radiation and skin, SaintVincent, 12-15 sept. 1981. S.I. : Associazione italiana radioprotezione medica, 1981, 99-131.

[23] YOUNG C.M.A., HOPEWELL J.W. The evaluation of an isotope clearence technique in the dermis of the pig skin : a correlation of functional and morphological parameters. Microvasc. Res., 1980, 20, 182-194. 